OPEN ACCESS

Edited by:

Pietro Cipresso, Italian Auxological Institute (IRCCS), Italy

Reviewed by: Givago Silva Souza, Federal University of Pará, Brazil Fabio Lucidi, Sapienza University of Rome, Italy

*Correspondence: Giulio Vidotto giulio.vidotto@unipd.it

Specialty section: This article was submitted to Quantitative Psychology and Measurement, a section of the journal Frontiers in Psychology

Received: 21 August 2019 Accepted: 27 November 2019

Published: 17 December 2019

Citation:

Vidotto G, Anselmi P and Robusto E (2019) New Perspectives in Computing the Point of Subjective Equality Using Rasch Models. Front. Psychol. 10:2793. doi: 10.3389/fpsyg.2019.02793

\section{New Perspectives in Computing the Point of Subjective Equality Using Rasch Models}

\author{
Giulio Vidotto ${ }^{1 *}$, Pasquale Anselmi ${ }^{2}$ and Egidio Robusto ${ }^{2}$ \\ ${ }^{1}$ Department of General Psychology, University of Padua, Padova, Italy, ${ }^{2}$ Department of Philosophy, Sociology, Education \\ and Applied Psychology, University of Padua, Padova, Italy
}

In psychophysics, the point of subject equality (PSE) is any of the points along a stimulus dimension at which a variable stimulus (visual, tactile, auditory, and so on) is judged by an observer to be equal to a standard stimulus. Rasch models have been found to offer a valid solution for computing the PSE when the method of constant stimuli is applied in the version of the method of transitions. The present work provides an overview of the procedures for computing the PSE using Rasch models and proposes some new developments. An adaptive procedure is described that allows for estimating the PSE of an observer without presenting him/her with all stimuli pairs. This procedure can be particularly useful in those situations in which psychophysical conditions of the individuals require that the number of trials is limited. Moreover, it allows for saving time that can be used to scrutinize the results of the experiment or to run other experiments. Also, the possibility of using Rasch-based fit statistics for identifying observers who gave unexpected judgments is explored. They could be individuals who, instead of carefully evaluating the presented stimuli pairs, gave random, inattentive, or careless responses, or gave the same response to many consecutive stimuli pairs. Otherwise, they could be atypical and clinically relevant individuals who deserve further investigation. The aforementioned developments are implemented using procedures and statistics that are well established in the framework of Rasch models. In particular, computerized adaptive testing procedures are used for efficiently estimating the PSE of the observers, whereas infit and outfit mean-squares statistics are used for detecting observers who gave unexpected judgments. Results of the analyses carried out on simulated data sets suggest that the proposed developments can be used in psychophysical experiments.

Keywords: method of constant stimuli, method of transitions, point of subjective equality, Rasch models, computerized adaptive testing, infit, outfit

\section{INTRODUCTION}

In psychophysics, the point of subject equality (PSE) is any of the points along a stimulus dimension at which a variable stimulus (visual, tactile, auditory, and so on) is judged by an observer to be equal to a standard stimulus. When the method of constant stimuli (see, e.g., Laming and Laming, 1992) is used to measure the PSE, the observer is presented with a 
number $I$ of variable stimuli, each of which is denoted by $V S_{i}, i=1,2, \ldots, I$. The variable stimuli are placed at equal intervals along the physical continuum, and are chosen in such a way that the stimulus at the inferior extreme is perceived little more than $0-5 \%$ of the times it is presented, whereas a stimulus at the superior extreme is perceived a little less than $95-100 \%$ of the times. The variable stimuli are presented, one at a time and in random order, paired with a standard stimulus (SS). The number of presentations for each pair $\left(V S_{i}, S S\right)$ typically varies from 20 to 200. The observer judges each pair $\left(V S_{i}, S S\right)$ and says which of the two stimuli has a greater (or a fewer) quantity of the attribute under consideration (e.g., volume, roughness, loudness, and so on). The PSE is the value of a comparison stimulus that, for a particular observer, is equally likely to be judged as higher or lower than that of a standard stimulus (Guilford, 1954; Bock and Jones, 1968).

As an example of method of constant stimuli, let us consider an experiment of sound perception in which SS is a 50-decibel sound and the variable stimuli are $I=9$ sounds from 30 to 70 decibels, at the distance of 5 decibels one from the next (i.e., $V S=30,35,40,45,50,55,60,65,70$ decibels). Pairs of sounds are presented in succession, the former sound being the $S S$ and the latter sound being the VS. The subject is asked to report whether or not the second sound (the VS) is louder than the first sound (the SS). In the experiment at hand, sound loudness is the target attribute. The PSE is the level (in decibel) of a comparison stimulus at which this stimulus is judged by the observer to be as loud as SS.

When the method of constant stimuli is used, the classical solution for obtaining the PSE is the least square method (Müller, 1879). The proportion $P\left(V S_{i}>S S\right)$ of times in which $V S_{i}$ is judged higher than $S S$ is computed for each $V S_{i}$. Then, each $P\left(V S_{i}>S S\right)$ is transformed in the corresponding $z$-score $z_{i}$ by using the inverse of the cumulative normal function. Alternative and more recent solutions for obtaining the PSE are the weighted least square method (Urban, 1908) and the maximum likelihood procedure (Whittaker and Robinson, 1967).

In some cases, the experimenter cannot use the method of constant stimuli in the classical form. This is particularly true when effects of adaptation, habituation, and sensitization may occur. The greater the number of presentations, the higher the probability that these effects will influence the judgments. In these situations, the method of constant stimuli would be unsuitable. On the one hand, a drastic reduction in the presentation of stimuli would be necessary to reduce biases. On the other hand, a high number of presentations is necessary (especially when the number of observers is small) for the method of constant stimuli to produce good results.

One solution is to present each pair $\left(V S_{i}, S S\right)$ to each observer only once, as it happens in the method of transitions (Masin and Cavedon, 1970; Masin and Vidotto, 1982, 1984). A transition occurs when the comparative judgment of the pair $\left(V S_{i}, S S\right)$ is different from that of the pair $\left(V S_{i+1}, S S\right)$. In this case, it is possible to assume that the PSE of the observer takes place between $V S_{i}$ and $V S_{i+1}$. More details about the method of transitions, as well as examples of application can be found in Masin and Vidotto (1984) and Burro et al. (2011).
Rasch models have been found to offer a valid solution for computing the PSE when the method of constant stimuli is applied in the version of the method of transitions (Vidotto et al., 1996; Burro et al., 2011). Rasch models represent a family of psychometric models for creating measurements from categorical data. In these models, the probability of observing specified responses (e.g., correct/incorrect; yes/no; never/sometimes/often/ always) is modeled as a function of person and item parameters. These parameters pertain to the level of a quantitative latent trait possessed by a person or item, and their specific meaning relies on the subject of the assessment. In educational assessments, for instance, person parameters indicate the ability (or attainment level) of persons, and item parameters indicate the difficulty of items. In health status assessments, person parameters indicate the health of persons, and item parameters indicate the severity of items. The higher the ability of a person relative to the difficulty of an item, the higher the probability that the person will give a correct response to the item. The higher the health of a person relative to the severity of an item, the higher the probability that that person will give to the item a response that is indicative of health (e.g., a response "no" to an item like "I have trouble falling asleep"). Because of their general applicability, Rasch models have been used in several areas, including personality and health assessment, education, and market research (see, e.g., Bechtel, 1985; Vidotto et al., 1998, 2006, 2007, 2010a,b; Duncan et al., 2003; Cole et al., 2004; Bezruczko, 2005; Pallant and Tennant, 2007; Shea et al., 2009; Anselmi et al., 2011, 2013a,b, 2015; Da Dalt et al., 2013, 2015, 2017; Balsamo et al., 2014; Rossi Ferrario et al., 2019; Sotgiu et al., 2019).

When applied to psychophysics, Rasch models allow for identifying two aspects linked to the perceptive judgments. The first one deals with the ability of observers to discriminate the variable stimuli (parameters $\beta$ ). The second one deals with the difficulty of discriminating the variable stimuli from the standard stimulus (parameters $\delta$ ). These two types of parameters are placed on the same linear scale and can be compared (see, e.g., Andrich, 1988; Wright and Stone, 1999). The comparison between the discriminative ability of an observer and the discriminability of a variable stimulus allows for computing the probability that the observer will judge the variable stimulus in a certain way. It is worth noting that, within the Rasch framework, the estimates of observers' discriminative abilities do not depend on the specific collection of stimuli the observers have been presented with, as well as the estimates of stimuli' discriminability do not depend on the particular sample of observers who have been presented with the stimuli (Rasch, 1960; Bond and Fox, 2001).

There are algorithms that allow for estimating the parameters $\beta$ and $\delta$ from experimental data (see, e.g., Wright, 1977; Linacre, 1999; Wright and Stone, 1999), as well as procedures for deriving the PSE of an observer from his/her parameter $\beta$ (Vidotto et al., 1996; Burro et al., 2011). Moreover, there are Rasch models for simple judgments (the variable stimulus can only be considered to be higher or lower than the standard stimulus) and for more complex judgments (the variable stimulus can also be considered as not different from the standard stimulus). In particular, the simple logistic model (SLM, Rasch, 1960) is suitable in the first case, whereas the rating scale 
model (RSM; Andrich, 1978) is suitable in the second case. An application of the RSM for computing the PSE in a psychophysical experiment with three response categories is described in Burro et al. (2011).

The present work provides an overview of the procedures for computing the PSE using Rasch models. Besides, it proposes two new developments that are based on Rasch models and that pertain to the efficient estimation of the PSE and the identification of observers with unexpected judgments. Concerning the first development, a computerized adaptive testing (CAT) procedure is described that allows for estimating the PSE of an observer without presenting him/her with all stimuli pairs. This procedure can be particularly useful in those situations in which psychophysical conditions of individuals require that the number of trials is limited. Moreover, it allows for saving time that can be used to scrutinize the results of the experiment or to run other experiments. Concerning the second development, the possibility of using fit statistics for identifying observers who gave unexpected judgments is explored. They could be individuals who, instead of carefully evaluating the presented stimuli pairs, gave random, inattentive, or careless responses, or gave the same response to many consecutive stimuli pairs. Otherwise, they could be atypical and clinically relevant individuals for whom a further investigation is needed. The aforementioned developments are implemented using procedures and statistics that are well established in the framework of Rasch models and their functioning is illustrated via simulated data.

\section{COMPUTING THE POINT OF SUBJECTIVE EQUALITY USING RASCH MODELS}

Vidotto et al. (1996) and Burro et al. (2011) proposed to use Rasch models for computing the PSE of observers when the method of constant stimuli is applied in the version of the method of transitions. The authors focused on two models, namely the SLM and the RSM. The former is meant for dichotomous outcomes. As such, it is suitable for psychophysical experiments with two response categories (i.e., in which the variable stimulus can only be considered to be higher or lower than the standard stimulus). The RSM is an extension of the SLM meant for polytomous outcomes. As such, it is suitable for psychophysical experiments with more than two response categories (i.e., in which the variable stimulus can also be considered as not different from the standard stimulus).

Let $x_{n i}$ be the perceptive outcome obtained by observer $n$ in relation to the comparison between $V S_{i}$ and $S S$. If the observer $n$ can only report which of the two stimuli has a greater or a smaller quantity of the target attribute, then $x_{n i}$ assumes value 1 if $V S_{i}$ is perceived higher than $S S$, and value 0 if it is perceived lower than SS. If the observer $n$ is allowed to say that the two stimuli have the same quantity of the target attribute, then $x_{n i}$ assumes value 2 if $V S_{i}$ is perceived higher than $S S$, value 1 if $V S_{i}$ and $S S$ are perceived as equal, and value 0 if $V S_{i}$ is perceived lower than $S S$.
For instance, let us still consider the experiment of sound perception in which pairs of sounds are presented in succession, and the subject is asked to report whether or not the second sound (VS $=30,35,40,45,50,55,60,65,70$ ) is louder than the first sound ( $S S=50$ decibels). Table 1 shows possible perceptive outcomes for experimental situations with two or three response options. In the former situation, the variable stimuli of $30,35,40,45,50,60$ decibels are judged to be less loud than SS, and those of 55, 65, and 70 decibels are judged to be louder than SS. In the latter situation, the variable stimuli of $30,35,40,45,55$ decibels are judged to be less loud than SS; those of 50 and 60 decibels are judged to be as loud as SS; and those of 65 and 70 decibels are judged to be louder than SS.

It is worth noting that sometimes the response option of equal judgments does not actually mean that the two stimuli are perceived as having the same quantity of target attribute but it takes the meaning of "I do not know", "I am uncertain about," or "It seems to me that they are different but I am not sure which one is the greatest."

The SLM and the RSM describe the probability of observing the perceptive outcome $x_{n i}$ as:

$$
P\left(X_{n i}=x_{n i} \mid \beta_{n}, \delta_{i}\right)=\frac{\exp \left(x_{n i}\left(\beta_{n}-\delta_{i}\right)\right)}{1+\exp \left(\beta_{n}-\delta_{i}\right)},
$$

and

$$
P\left(X_{n i}=x_{n i} \mid \beta_{n}, \delta_{i}, \tau_{k}\right)=\frac{\exp \sum_{k=0}^{x}\left(\beta_{n}-\left(\delta_{i}-\tau_{k}\right)\right)}{\sum_{j=0}^{m} \exp \sum_{k=0}^{j}\left(\beta_{n}-\left(\delta_{i}-\tau_{k}\right)\right)},
$$

where:

1. $\beta_{n}$ is the discriminative ability of observer $n$;

2. $\delta_{i}$ is the difficulty of discriminating the variable stimulus $V S_{i}$ from the standard stimulus $S S$;

TABLE 1 | Example of perceptive outcomes in an experiment of sound perception with $S S$ of 50 decibels.

Perceptive outcome

\begin{tabular}{ccc}
\hline $\boldsymbol{v} \boldsymbol{s}_{\boldsymbol{i}}$ (decibels) & Two response options & Three response options \\
\hline 30 & 0 & 0 \\
35 & 0 & 0 \\
40 & 0 & 0 \\
45 & 0 & 0 \\
50 & 0 & 1 \\
55 & 1 & 0 \\
60 & 0 & 1 \\
65 & 1 & 2 \\
70 & 1 & 2 \\
\hline
\end{tabular}

In the condition with two response options, the perceptive outcome takes the values 0

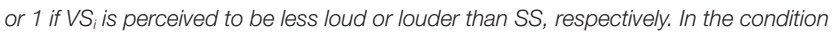
with three response options, the perceptive outcome takes the values 0,1 , or 2 if V $S_{j}$ is perceived to be less loud than, as loud as, or louder than SS, respectively. 
3. $\tau_{k}$ is the $k$-th threshold and expresses the passage from one response category to the next one (thus, if the measurement criterion includes three response categories, there will be two thresholds).

Once parameters $\beta$ and $\delta$ have been estimated, the PSE of observer $n$ is obtained through the following steps:

1. The difficulties of stimuli $\left(\delta_{i}\right)$ are put in relation to the relative physical values $\varphi_{i}$. This determines the intercept and the slope of the regression line (i.e., $\varphi_{i}=a \delta_{i}+b$ ).

2. The obtained values of intercept and slope are used to derive the PSEs of observers from their discriminative abilities (i.e., $\operatorname{PSE}_{n}=a \beta_{n}+b$ ).

\section{AN ADAPTIVE PROCEDURE FOR ESTIMATING THE POINT OF SUBJECTIVE EQUALITY}

One of the most prominent applications of Rasch models is in CAT. CAT procedures allow for accurately estimating the latent trait level of individuals by presenting them with only a minimum number of items (Linacre, 2000). Typically, the adaptive tests reach the same level of accuracy of the conventional fixed-length tests using about 50\% of the items (Embretson and Reise, 2000; van der Linden, 2008). Moreover, the adaptive tests can be a better experience for individuals, as they are only presented with items targeted at their level (Deville, 1993). This section describes the functioning of a CAT procedure that aims at estimating the PSE of an observer.

CAT is preceded by a preliminary phase in which the psychophysical experiment is run on a suitable calibration sample, and an appropriate Rasch model (either the SLM or the RSM) is estimated on the collected data. This phase aims to arrive at an accurate estimate of the parameters $\delta$ (if the SLM is estimated) or $\delta$ and $\tau$ (if the RSM is estimated), so that they can be considered as known during CAT. When the latter begins, the only unknown parameters are the discriminative abilities $\beta$ of observers under evaluation.

Figure 1 depicts the functioning of the CAT procedure. An initial estimate is determined for the discriminative ability $\beta$ of the observer. The first pair $\left(V S_{i}, S S\right)$ is selected based on this starting point and presented to the observer. The pair is judged and scored, and the estimate of $\beta$ is updated accordingly. The stopping criterion is then evaluated. If it is not yet satisfied, another pair $\left(V S_{i}, S S\right)$ is selected based on the current estimate $\beta$. The observer judges this new pair, and the estimate of $\beta$ is updated again. The procedure iterates the aforementioned steps until the stopping criterion is satisfied.

There are several methods and algorithms for implementing each of the steps in a CAT procedure. A brief overview of the main ones is presented here. Readers interested in a more comprehensive discussion are referred to, for instance, Linacre (2000), van der Linden and Glas (2000), Wainer et al. (2000), van der Linden and Pashley (2010), and Thompson and Weiss (2011).

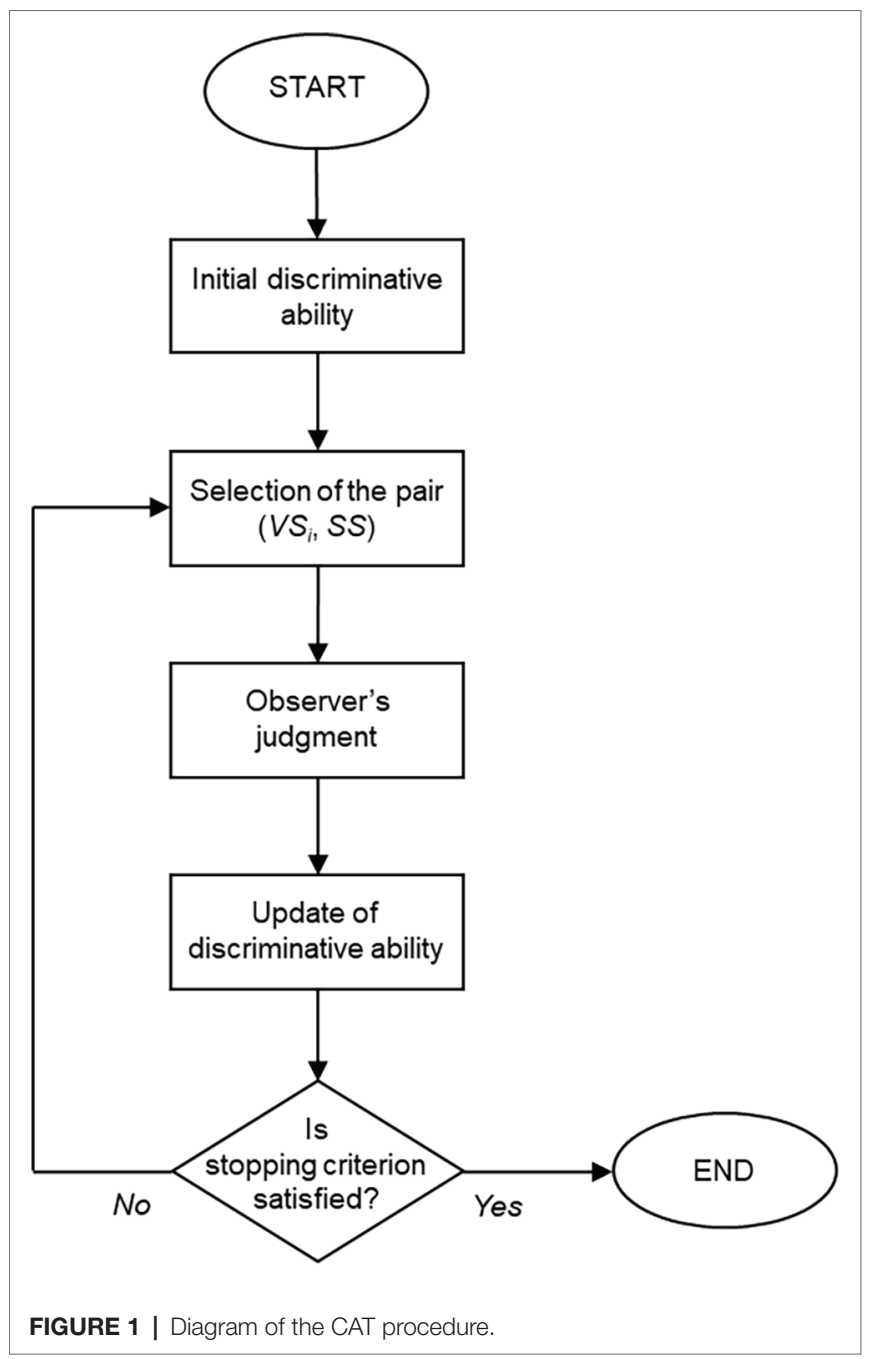

Determination of the initial estimate for the discriminative ability: Different options are available for this purpose. The most straightforward one is to use, as an initial estimate of observer's discriminative ability, the mean of the $\beta$ distribution obtained on the calibration sample. Otherwise, if the information on the observer is available (e.g., results of a previous psychophysical experiment, familiarity of the observer with the perceptive task under consideration), this information can be used to determine a more appropriate initial estimate.

Selection of the pair $\left(V S_{i}, S S\right)$ to be presented: The idea is to select the pair $\left(V S_{i}, S S\right)$ according to the observer's estimated discriminative ability. A method very common in traditional CAT would imply to select the pair that maximizes Fisher information at the current estimate of discriminative ability. This method allows for estimating observer's discriminative ability by presenting him/her with a minimum number of stimuli pairs.

Update of observer's discriminative ability: The current estimate of the observer's discriminative ability is updated based on his/her response to the latest administered stimuli pair. Common methods are maximum-likelihood and Bayesian methods such 
as expected a posteriori (EAP, Bock and Mislevy, 1988) and maximum a posteriori (MAP, Mislevy, 1986).

Stopping criterion: CAT can be designed to be either fixedlength or variable-length. In the former case, the procedure stops when a specified number of stimuli pairs has been presented. In the second case, the procedure can stop when observer's $\beta$ estimate changes below a certain small amount from one iteration to the other or has reached a certain level of precision, or when no stimuli pairs are left that provide at least some minimal level of information.

\section{Method \\ Data Simulation}

A psychophysical experiment with 11 variable stimuli was considered (i.e., $I=11$ ). The stimuli were placed at the distance of one unit along the physical continuum. The smallest variable stimulus was five units smaller than the SS, whereas the largest variable stimulus was five units larger than the SS. A condition was simulated in which the observers judged each pair $\left(V S_{i}\right.$, SS) and reported which of the two stimuli of the pair was the highest (two response options).

Two data samples of 100 observers each were simulated. One sample was used as a calibration sample, the other sample was used for running the CAT procedure (CAT sample). For both samples, 100 PSE values were randomly drawn from a normal distribution with mean $=-1.5$ and standard deviation $=1$.

\section{Calibration and Computerized Adaptive Testing}

The SLM was estimated on the calibration sample. Model parameters were estimated using the EAP method.

The CAT procedure was run on the CAT sample using the estimates of parameters $\delta$ that were obtained on the calibration sample. The mean of the $\beta$ distribution obtained on the calibration sample was used as initial estimate of observer's discriminative ability in the CAT procedure. Maximum Fisher information was used for selecting the stimuli pair to the administered. The responses to the selected stimuli pairs were extracted from the CAT sample. The EAP method was used for updating the estimates of $\beta$. For each observer in the CAT sample, the estimates of $\beta$ and PSE were computed for the first five stimuli pairs that were presented.

The performance of the CAT procedure was compared with that of a procedure in which, at each iteration, the stimuli pair to be presented was randomly chosen (random procedure).

\section{Results}

Table 2 shows the estimates of parameters $\delta$ that were obtained on the calibration sample.

Figure 2 depicts the results of the CAT and random procedures. The left diagram depicts the average absolute difference between the parameters $\beta$ estimated after the presentation of a certain number of stimuli pairs (from 1 to 5 pairs) and those estimated on all stimuli pairs (11 pairs). The right diagram depicts the average absolute difference between the PSEs estimated after the presentation of a certain number of stimuli pairs and those estimated on all stimuli pairs. In
TABLE 2 | Estimates of parameters $\delta$ obtained on the calibration sample.

\begin{tabular}{ccc}
\hline $\begin{array}{c}\text { Difference between } \\
\boldsymbol{V} \boldsymbol{S}_{\boldsymbol{i}} \text { and } \boldsymbol{S S}\end{array}$ & $\boldsymbol{\delta}$ & $\boldsymbol{S E}$ \\
\hline-5 & & \\
\hline-4 & -2.45 & 0.33 \\
-3 & -2.82 & 0.37 \\
-2 & -2.34 & 0.32 \\
-1 & -1.97 & 0.29 \\
0 & -0.67 & 0.23 \\
1 & 0.85 & 0.24 \\
2 & 1.38 & 0.25 \\
3 & 2.33 & 0.32 \\
4 & 2.33 & 0.32 \\
5 & 2.55 & 0.34 \\
& 1.97 & 0.29 \\
\hline
\end{tabular}

both diagrams, the solid line denotes the CAT procedure, the dashed line denotes the random procedure. The bars denote 95\% confidence intervals. For both CAT and random procedures, with the increasing of the number of presented stimuli pairs, the estimates of $\beta$ and PSE approach those obtained on all stimuli pairs. However, the number of presented pairs being equal, the CAT procedure outperforms the random procedure in approximating the estimates obtained on all stimuli pairs. The differences between the estimates $\beta$ and PSE obtained on 4 or 5 stimuli pairs and those obtained on all stimuli pairs are significantly smaller when stimuli pairs are selected by the CAT procedure, rather than by the random procedure.

Figure 3 depicts the correlation between the PSEs estimated after the presentation of a certain number of stimuli pairs and those estimated on all stimuli pairs. The solid line denotes the CAT procedure, the dashed line denotes the random procedure. For both CAT and random procedures, the strength of the correlation between the PSEs estimated on the presented stimuli pairs and those estimated on all stimuli pairs increases with the number of presented stimuli pairs. On the whole, the number of presented stimuli pairs being equal, the correlation is significantly stronger when PSEs are estimated on the stimuli pairs selected by the CAT procedure than on those selected by the random procedure $(z \geq 1.98, p<0.05$ when $1,3,4$, or 5 stimuli pairs are presented; $z=1.21, p=0.23$ when 2 stimuli pairs are presented).

Results of this simulation study suggest that a Rasch-based CAT procedure can be used for estimating the PSE of observers without presenting them with all stimuli pairs.

\section{IDENTIFICATION OF OBSERVERS WHO GAVE UNEXPECTED JUDGMENTS}

Rasch framework provides infit and outfit mean-square statistics that allow for detecting individuals with unexpected response behaviors. For instance, these statistics have been used to identify possible fakers to self-report personality tests (Vidotto et al., 2018) and to identify individuals who miss items they are not capable of solving (Anselmi et al., 2018). This section explores the use of these statistics in psychophysical experiments 

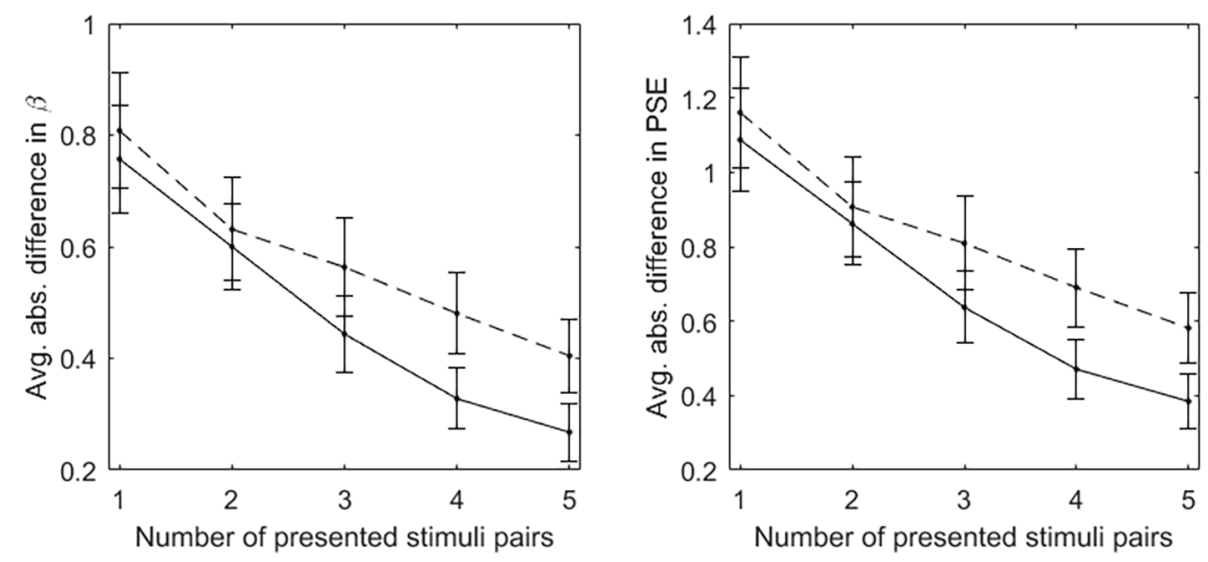

FIGURE 2 | Results of CAT (solid line) and random (dashed line) procedures. The left diagram depicts the average absolute difference between the parameters $\beta$ estimated after the presentation of a certain number of stimuli pairs and those estimated on all stimuli pairs. The right diagram depicts the average absolute difference between the PSEs estimated after the presentation of a certain number of stimuli pairs and those estimated on all stimuli pairs. The bars denote 95\% confidence intervals.

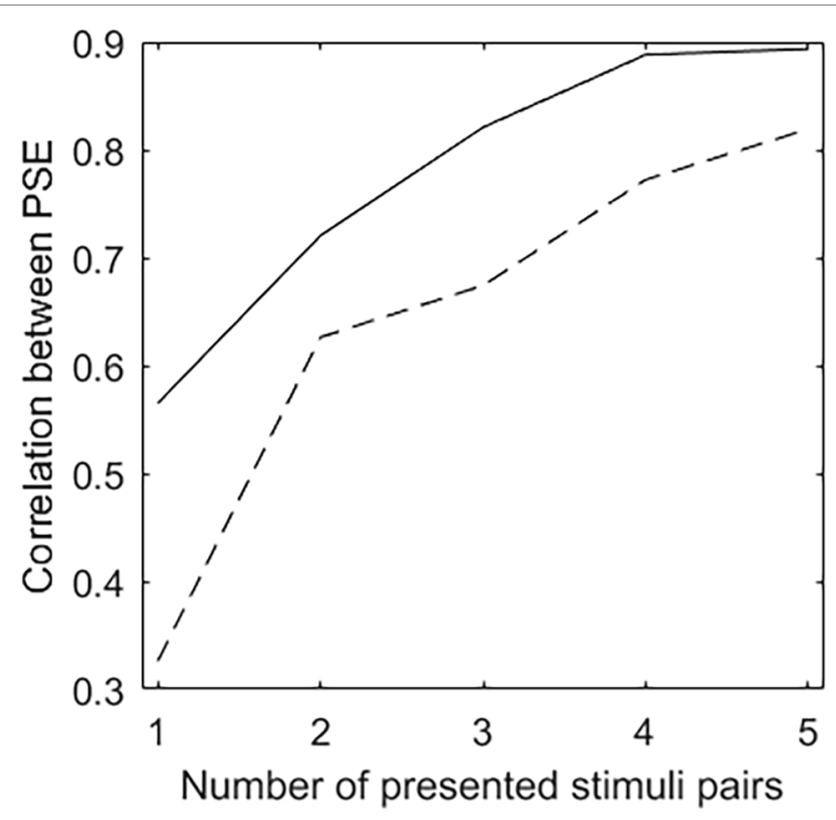

FIGURE 3 | Correlation between the PSEs estimated after the presentation of a certain number of stimuli pairs and those estimated on all stimuli pairs. The solid line denotes the CAT procedure, the dashed line denotes the random procedure.

to identify observers who gave unexpected judgments. They could be individuals who, instead of carefully evaluating the presented stimuli pairs, gave random, inattentive or careless responses, or gave the same response to many consecutive stimuli pairs. Otherwise, they could be atypical and clinically relevant individuals who deserve further investigation.

Infit and outfit mean-square statistics are $\chi^{2}$ statistics divided by their degrees of freedom, with an expected value of 1 . Values greater than 1 for an observer indicate that his/her judgments are less predictable than the Rasch model expects.
Infit is influenced more by slightly unexpected judgments (i.e., those observed when the discriminative ability of the observer is similar to the difficulty of the variable stimulus to be discriminated). Outfit is influenced more by highly unexpected judgments (i.e., those observed when the discriminative ability of the observer is quite different from the difficulty of the variable stimulus to be discriminated). Observers with infit or outfit above a certain, appropriately chosen cut-off are flagged as possible observers with careless or random judgments and removed from the data set. A common choice for the cut-off is 1.5 (Wright and Linacre, 1994; Linacre, 2002).

\section{Methods \\ Data Simulation}

A psychophysical experiment with 11 variable stimuli at the distance of one unit from each other was considered. The smallest variable stimulus was five units smaller than the SS, whereas the largest variable stimulus was five units larger than the SS. A condition was simulated in which the observers reported which of the two stimuli of each pair $\left(V S_{i}, S S\right)$ was the highest.

One data sample of 100 observers was simulated, by randomly drawing 100 PSE values from a normal distribution with mean $=-1.5$ and standard deviation $=1$. This data set is denoted as the original data set. Ten of the observers in the original data set were randomly selected and their judgments to six stimuli pairs, randomly chosen among the 11 pairs, were set to be different from those in the original data set. This data set is denoted as the noisy data set.

The SLM was estimated on the two data sets. EAP estimates of the parameters $\beta$ and $\delta$ were computed.

\section{Results}

The PSEs were estimated with the Rasch model and with the method of transitions (Masin and Vidotto, 1984; Burro et al., 2011). In what follows, the former are denoted as Rasch-PSEs and the latter are denoted as transition-PSEs. 
The Rasch-PSEs estimated on the original data set $(M=-1.30$, $s=1.69$ ) do not differ from the randomly drawn true PSEs $(M=-1.50 ; s=1.00)[t(99)=-1.95, p=0.05$, Cohen's $d=-0.15$, Pearson's $r=0.78]$, whereas the transition-PSEs $(M=-1.27$; $s=1.48)$ differ $[t(99)=-2.60, p<0.05$, Pearson's $r=0.78]$ although the effect size is small (Cohen's $d=-0.19$ ).

Both Rasch-PSEs and transition-PSEs estimated on the noisy data set differ from the randomly drawn true PSEs [RaschPSEs: $M=-1.02, s=1.78, t(99)=-3.49, p<0.001$, Cohen's $d=-0.33$, Pearson's $r=0.63$; transition-PSEs: $M=-1.03$, $s=1.58, t(99)=-3.85, p<0.001$, Cohen's $d=-0.35$, Pearson's $r=0.62]$.

Sensitivity and specificity of the cut-off at 1.5 were computed for both fit statistics (infit, outfit) that were obtained for each of the 100 observers in the noisy data set. Sensitivity refers to the capacity of correctly detecting observers with random judgments. It is the proportion of observers with fit statistic larger than 1.5 among those observers with random judgments. Specificity refers to the capacity of correctly ignoring observers without random judgments. It is the proportion of observers with fit statistic smaller than or equal to 1.5 among those observers without random judgments.

As regards outfit, the cut-off allowed for correctly identifying 8 of the 10 observers with random judgments (sensitivity $=0.80$ ) and for correctly ignoring 86 of the 90 observers without random responses (specificity $=0.96$ ). As regards infit, the cut-off allowed for correctly identifying 7 of the 10 observers with random judgments (sensitivity $=0.70$ ) and for correctly ignoring 87 of the 90 observers without random responses (specificity $=0.97)$.

A "cleaned" data set has been obtained by removing from the noisy data set the observers with the outfit above the cut-off. Both Rasch-PSEs and transition-PSEs estimated on the cleaned data set differ from the randomly drawn true PSEs (Rasch-PSEs: $M=-1.11, s=1.76, t(87)=-2.73$, $p<0.01$, Cohen's $d=-0.25$, Pearson's $r=0.70$; transitionPSEs: $M=-1.10, s=1.59, t(87)=-3.85, p<0.01$, Cohen's $d=-0.28$, Pearson's $r=0.70)$. However, the effect size of the difference between the true PSEs and those estimated on the cleaned data set is slightly smaller than that of the difference between the true PSEs and those estimated on the noisy data set (Rasch-PSEs: Cohen's $d=-0.25,-0.33$, respectively; transition-PSEs: Cohen's $d=-0.28,-0.35$, respectively). A similar result is obtained if the observers with the infit above the cut-off are removed [Rasch-PSEs: $M=-1.06, s=1.79, t(89)=-3.12, p<0.01$, Pearson's $r=0.68$, Cohen's $d=-0.29$ vs. -0.33 ; transition-PSEs: $M=-1.09, s=1.62, t(89)=-3.22, p<0.01$, Pearson's $r=0.68$, Cohen's $d=-0.30$ vs. -0.35$]$.

In all aforementioned conditions, correlations between RaschPSEs and transition-PSEs are very strong (Pearson's $r \geq 0.97$ ) and effect sizes of the differences are small (Cohen's $d \leq 0.19$ ).

Results of this simulation study suggest that Rasch-based infit and outfit statistics might allow the detection of observers with unexpected judgments. If these observers are removed from the data set, a more accurate estimate of the overall PSE is obtained.

\section{DISCUSSION}

The present work provided an overview of the procedures for computing the PSE using Rasch models and proposed two new developments that are based on procedures and statistics well-established in the framework of Rasch models.

A CAT procedure has been described that allows for estimating the PSE of observers without presenting them with all stimuli pairs. Each observer is asked to judge only those stimuli pairs that are most informative about his/her PSE. The method of transitions requires presenting all stimuli pairs. As such, it cannot be used for adaptively estimating the PSE of observers. Other procedures are available in psychophysical research that can be used for this purpose. The adaptive procedures that currently enjoy widespread use may be placed into three general categories, called parameter estimation by sequential testing, maximum-likelihood adaptive procedures, and staircase procedures (Treutwein, 1995; Leek, 2001). These procedures and that described in the present work share the goal of preserving the accuracy of measurement while maximizing efficiency and minimizing observer and experimenter time.

Infit and outfit have been shown to allow the identification of observers with unexpected judgments. The judgments expressed by each of these observers must be carefully analyzed to try to find out if they are clinically relevant individuals or people who simply performed the task without due attention. Individuals may be distracted during the experiment and forget about the intensity of the stimuli after the presentation, or completely miss them, resulting in biased or random responses (Rinderknecht et al., 2018). In psychophysical experiments, inattentive responses can be identified in at least two ways. Experienced experimenters may be able to potentially detect courses of performance being visibly influenced by inattention, based on sudden performance level decreases for a certain period. However, this way of analyzing the data is not reproducible (Rinderknecht et al., 2018). Physiological signals such as electrodermal activity could potentially be used to detect inattention intervals, as arousal has been found to be a strong predictor for attention (Prokasy and Raskin, 1973). However, the measurement of electrodermal activity requires additional equipment and may not be applicable in some experimental settings. The method described in this study might allow the identification of inattentive or random responses. The strengths of this method are its reproducibility and the fact that it is based solely on the responses recorded during the experiment. Within the method of transitions, no procedure has been developed for identifying observers with unexpected judgments. A possibility in this direction could be sorting the perceptive outcomes according to the physical levels of the variable stimuli and then counting the number of runs (each of which being a sequence of equal perceptive outcomes). A large number of runs might be indicative of observers with unexpected judgments.

It is worth noting that, once the Rasch model has been estimated and validated on a suitable sample of observers, it can be used for adaptively estimating the PSE of new observers, as well as for computing their infit and outfit statistics without having to re-estimate the model parameters. 


\section{Limitations and Suggestions for Future Research}

In the present work, the adaptive estimation of observers' PSEs and the detection of observers with unexpected judgments have been investigated via simulated data. A definitive advantage of using simulated data lies in the full knowledge of the data under consideration. Future works should investigate the usefulness of the proposed developments on real data resulting from psychophysical experiments.

In the present work, a basic Rasch-based CAT procedure has been implemented. However, the literature on CAT is rich in alternative methods that could be used for determining the starting point, selecting the stimuli pairs to be presented, updating the estimate of observer's discriminative ability, and stopping the procedure (see, e.g., Linacre, 2000; van der Linden and Glas, 2000; Wainer et al., 2000; van der Linden and Pashley, 2010; Thompson and Weiss, 2011). Future works should investigate the usefulness of these methods in psychophysical experiments and compare them with the adaptive procedures that are commonly used in psychophysical research (i.e., parameter estimation by sequential testing, maximum-likelihood adaptive procedures, staircase procedures).

In the present work, unexpected judgments have been simulated by randomly modifying the responses of some observers to some stimuli pairs. Other unexpected behaviors could be observed in psychophysical experiments (e.g., some observers could give the same response to many consecutive

\section{REFERENCES}

Andrich, D. (1978). A rating scale formulation for ordered response categories. Psychometrika 43, 561-573. doi: 10.1007/BF02293814

Andrich, D. (1988). Rasch models for measurement. Beverly Hills: Sage.

Anselmi, P., Robusto, E., and Cristante, F. (2018). Analyzing missingness at the level of the individual respondent: comparison of five statistical tests. Test. Psychom. Methodol. Appl. Psychol. 25, 379-394. doi: 10.4473/TPM25.3.4

Anselmi, P., Vianello, M., and Robusto, E. (2011). Positive associations primacy in the IAT. A many-facet Rasch measurement analysis. Exp. Psychol. 58, 376-384. doi: 10.1027/1618-3169/a000106

Anselmi, P., Vianello, M., and Robusto, E. (2013a). Preferring thin people does not imply derogating fat people. A Rasch analysis of the implicit weight attitude. Obesity 21, 261-265. doi: 10.1002/oby.20085

Anselmi, P., Vianello, M., Voci, A., and Robusto, E. (2013b). Implicit sexual attitude of heterosexual, gay and bisexual individuals: disentangling the contribution of specific associations to the overall measure. PLoS One 8:e78990. doi: 10.1371/journal.pone.0078990

Anselmi, P., Vidotto, G., Bettinardi, O., and Bertolotti, G. (2015). Measurement of change in health status with Rasch models. Health Qual. Life Outcomes 13:16. doi: 10.1186/s12955-014-0197-x

Balsamo, M., Giampaglia, G., and Saggino, A. (2014). Building a new Raschbased self-report inventory of depression. Neuropsychiatr. Dis. Treat. 10, 153-165. doi: 10.2147/NDT.S53425

Bechtel, G. G. (1985). Generalizing the Rasch model for consumer rating scales. Mark. Sci. 4, 62-73. doi: 10.1287/mksc.4.1.62

Bezruczko, N. (2005). Rasch measurement in health sciences. Maple Grove, MN: Jam Press.

Bock, R. D., and Jones, L. V. (1968). The measurement and prediction of judgment and choice. San Francisco: Holden-Day.

Bock, R. D., and Mislevy, R. J. (1988). Adaptive EAP estimation of ability in a microcomputer environment. Appl. Psychol. Meas. 6, 431-444. doi: 10.1177/ 014662168200600405 stimuli pairs). Moreover, in the present work, a single cut-off at 1.5 has been used. Future work could explore the usefulness of infit and outfit statistics to detect different types of response behaviors when various cut-offs are employed.

\section{DATA AVAILABILITY STATEMENT}

The $\mathrm{R}$ scripts used for simulating and analyzing the data will be made available by the authors, without undue reservation, to any qualified researcher.

\section{AUTHOR CONTRIBUTIONS}

GV and PA contributed to the conception and design of the study. PA performed the statistical analyses and wrote the first draft of the manuscript. All authors contributed to manuscript revision, read and approved the submitted version.

\section{FUNDING}

The present work was carried out within the scope of the research program "Dipartimenti di Eccellenza," which is supported by a grant from MIUR to the Department of General Psychology, University of Padua.

Bond, T. G., and Fox, C. M. (2001). Applying the Rasch model: Fundamental measurement in the human sciences. Mahwah: Lawrence Erlbaum.

Burro, R., Sartori, R., and Vidotto, G. (2011). The method of constant stimuli with three rating categories and the use of Rasch models. Qual. Quant. 45, 43-58. doi: 10.1007/s11135-009-9282-3

Cole, J. C., Rabin, A. S., Smith, T. L., and Kaufman, A. S. (2004). Development and validation of a Rasch-derived CES-D short form. Psychol. Assess. 16, 360-372. doi: 10.1037/1040-3590.16.4.360

Da Dalt, L., Anselmi, P., Bressan, S., Carraro, S., Baraldi, E., Robusto, E., et al. (2013). A short questionnaire to assess pediatric resident's competencies: the validation process. Ital. J. Pediatr. 39:41. doi: 10.1186/1824-7288-39-41

Da Dalt, L., Anselmi, P., Furlan, S., Carraro, S., Baraldi, E., Robusto, E., et al. (2015). Validating a set of tools designed to assess the perceived quality of training of pediatric residency programs. Ital. J. Pediatr. 41:2. doi: 10.1186/ s13052-014-0106-2

Da Dalt, L., Anselmi, P., Furlan, S., Carraro, S., Baraldi, E., Robusto, E., et al. (2017). An evaluation system for postgraduate pediatric residency programs: report of a 3-year experience. Eur. J. Pediatr. 176, 1279-1283. doi: 10.1007/ s00431-017-2967-z

Deville, C. (1993). Flow as a testing ideal. Rasch Meas. Trans. 7:308.

Duncan, P. W., Bode, R. K., Lai, S. M., and Perera, S.Glycine Antagonist in Neuroprotection Americas Investigators (2003). Rasch analysis of a new stroke-specific outcome scale: the stroke impact scale. Arch. Phys. Med. Rehabil. 84, 950-963. doi: 10.1016/S0003-9993(03)00035-2

Embretson, S., and Reise, S. (2000). Item response theory for psychologists. Mahwah, NJ: Erlbaum.

Guilford, J. P. (1954). Psychometric methods. New York: McGraw-Hill.

Laming, D., and Laming, J. (1992). F. Hegelmaier: on memory for the length of a line. Psychol. Res. 54, 233-239. doi: 10.1007/BF01358261

Leek, M. R. (2001). Adaptive procedures in psychophysical research. Percept. Psychophys. 63, 1279-1292. doi: 10.3758/BF03194543

Linacre, J. M. (1999). Understanding Rasch measurement: estimation methods for Rasch measures. J. Outcome Meas. 3, 382-405. 
Linacre, J. M. (2000). "Computer-adaptive testing: a methodology whose time has come" in Development of computerized middle school achievement test. eds. S. Chae, U. Kang, E. Jeon, and J. M. Linacre (Seoul, South Korea: Komesa Press). Available at: https://www.rasch.org/memo69.pdf

Linacre, J. M. (2002). What do infit and outfit, mean-square and standardized mean? Rasch Meas. Trans. 16, 878.

Masin, S. C., and Cavedon, A. (1970). The estimation of the point of subjective equality and its standard error by averaging equals and transitions from 'greater' of 'less' in the method of constant stimuli. A preliminary investigation. Ita. J. Psychol. 7, 183-186.

Masin, S. C., and Vidotto, G. (1982). A review of the formulas for the standard error of a threshold from the method of constant stimuli. Percept. Psychophys. 31, 585-588. doi: 10.3758/BF03204194

Masin, S. C., and Vidotto, G. (1984). The method of transitions. Percept. Psychophys. 36, 593-594. doi: 10.3758/BF03207521

Mislevy, R. J. (1986). Bayes modal estimation in item response models. Psychometrika 51, 177-195. doi: 10.1007/BF02293979

Müller, G. E. (1879). Über die massbestimmungen des ortssinmes der haut mittels der methode der richtigen und falschen fälle. Pflugers Arch. 19, 191-235. doi: 10.1007/BF01639850

Pallant, J. F., and Tennant, A. (2007). An introduction to the Rasch measurement model: an example using the hospital anxiety and depression scale (HADS). Br. J. Clin. Psychol. 46, 1-18. doi: 10.1348/014466506X96931

Prokasy, W. F., and Raskin, D. C. (eds) (1973). Electrodermal activity in psychological research. New York: Academic Press.

Rasch, G. (1960). Probabilistic models for some intelligence and attainment test. Copenhagen: Danish Institute for Educational Research. Reprinted, 1980. Chicago: The University of Chicago Press.

Rinderknecht, M. D., Ranzani, R., Popp, W. L., Lambercy, O., and Gassert, R. (2018). Algorithm for improving psychophysical threshold estimates by detecting sustained inattention in experiments using PEST. Atten. Percept. Psychophys. 80, 1629-1645. doi: 10.3758/s13414-018-1521-Z

Rossi Ferrario, S., Panzeri, A., Anselmi, P., and Vidotto, G. (2019). Development and psychometric properties of a short form of the illness denial questionnaire. Psychol. Res. Behav. Manag. 12, 727-739. doi: 10.2147/PRBM.S207622

Shea, T. L., Tennant, A., and Pallant, J. F. (2009). Rasch model analysis of the depression, anxiety and stress scales (DASS). BMC Psychiatry 9:21. doi: 10.1186/1471-244X-9-21

Sotgiu, I., Anselmi, P., and Meneghini, A. M. (2019). Investigating the psychometric properties of the questionnaire for Eudaimonic well-being: a Rasch analysis. Test. Psychom. Methodol. Appl. Psychol. 26, 237-247. doi: 10.4473/TPM26.2.5

Thompson, N. A., and Weiss, D. J. (2011). A framework for the development of computerized adaptive tests. Pract. Assess. Res. Eval. 16, 1-9.

Treutwein, B. (1995). Adaptive psychophysical procedures. Vis. Res. 35, 2503-2522. doi: 10.1016/0042-6989(95)00016-X

Urban, F. M. (1908). The application of statistical methods to the problems of psychophysics. Philadelphia, PA: The Psychological Clinic Press.

van der Linden, W. J. (2008). Some new developments in adaptive testing technology. J. Psychol. 216, 3-11. doi: 10.1027/0044-3409.216.1.3

van der Linden, W. J., and Glas, C. A. W. (eds) (2000). Computerized adaptive testing: Theory and practice. Dordrecht, The Netherlands: Kluwer. van der Linden, W. J., and Pashley, P. J. (2010). "Item selection and ability estimation in adaptive testing" in Elements of adaptive testing. eds. W. J. van der Linden and C. A. W. Glas (New York: Springer), 3-30.

Vidotto, G., Anselmi, P., Filipponi, L., Tommasi, M., and Saggino, A. (2018) Using overt and covert items in self-report personality tests: susceptibility to faking and identifiability of possible fakers. Front. Psychol. 9:1100. doi: 10.3389/ fpsyg.2018.01100

Vidotto, G., Bertolotti, G., Carone, M., Arpinelli, F., Bellia, V., Jones, P. W., et al. (2006). A new questionnaire specifically designed for patients affected by chronic obstructive pulmonary disease; The Italian Health Status Questionnaire. Respir. Med. 100, 862-870. doi: 10.1016/j.rmed.2005.08.024

Vidotto, G., Carone, M., Jones, P. W., Salini, S., and Bertolotti, G. (2007). Maugeri respiratory failure questionnaire reduced form: a method for improving the questionnaire using the Rasch model. Disabil. Rehabil. 29, 991-998. doi: 10.1080/09638280600926678

Vidotto, G., Ferrario, S. R., Bond, T. G., and Zotti, A. M. (2010a). Family strain questionnaire - short form for nurses and general practitioners. J. Clin. Nurs. 19, 275-283. doi: 10.1111/j.1365-2702.2009.02965.x

Vidotto, G., Moroni, L., Burro, R., Filipponi, L., Balestroni, G., Bettinardi, O., et al. (2010b). A revised short version of the depression questionnaire. Eur. J. Cardiovasc. Prev. Rehabil. 17, 187-197. doi: 10.1097/HJR.0b013e328333edc8

Vidotto, G., Pegoraro, S., and Argentero, P. (1998). Modelli di Rasch e modelli di equazioni strutturali nella validazione del locus of control in ambito lavorativo. BPA Appl. Psychol. Bull. 227-228, 49-63.

Vidotto, G., Robusto, E., and Zambianchi, E. (1996). I modelli simple logistic e rating scale nella determinazione del punto di eguaglianza soggettivo: Una nuova prospettiva per il metodo degli stimuli costanti. Psychom. Methodol. Appl. Psychol. 3, 227-235.

Wainer, H., Dorans, N., Eignor, D., Flaugher, R., Green, B., and Mislevy, R. et al. (eds) (2000). Computerized adaptive testing: A primer. 2nd Edn. Mahwah, NJ: Erlbaum.

Whittaker, E. T., and Robinson, G. (1967). The calculus of observations: An introduction to numerical analysis. New York: Dover.

Wright, B. D. (1977). Solving measurement problems with the Rasch model. J. Educ. Meas. 14, 97-116. doi: 10.1111/j.1745-3984.1977.tb00031.x

Wright, B. D., and Linacre, J. M. (1994). Reasonable mean-square fit values. Rasch Meas. Trans. 8:370.

Wright, B., and Stone, M. (1999). Measurement essentials. 2nd Edn. Wilmington, DE: Wide Range, Inc.

Conflict of Interest: The authors declare that the research was conducted in the absence of any commercial or financial relationships that could be construed as a potential conflict of interest.

Copyright (c) 2019 Vidotto, Anselmi and Robusto. This is an open-access article distributed under the terms of the Creative Commons Attribution License (CC $B Y)$. The use, distribution or reproduction in other forums is permitted, provided the original author(s) and the copyright owner(s) are credited and that the original publication in this journal is cited, in accordance with accepted academic practice. No use, distribution or reproduction is permitted which does not comply with these terms. 